\title{
VARIATIONAL APPROACH TO CONICAL BODIES HAVING MAXIMUM LIFT-TO-DRAG RATIO AT HYPERSONIC SPEEDS
}

by

HO-YI HUANG

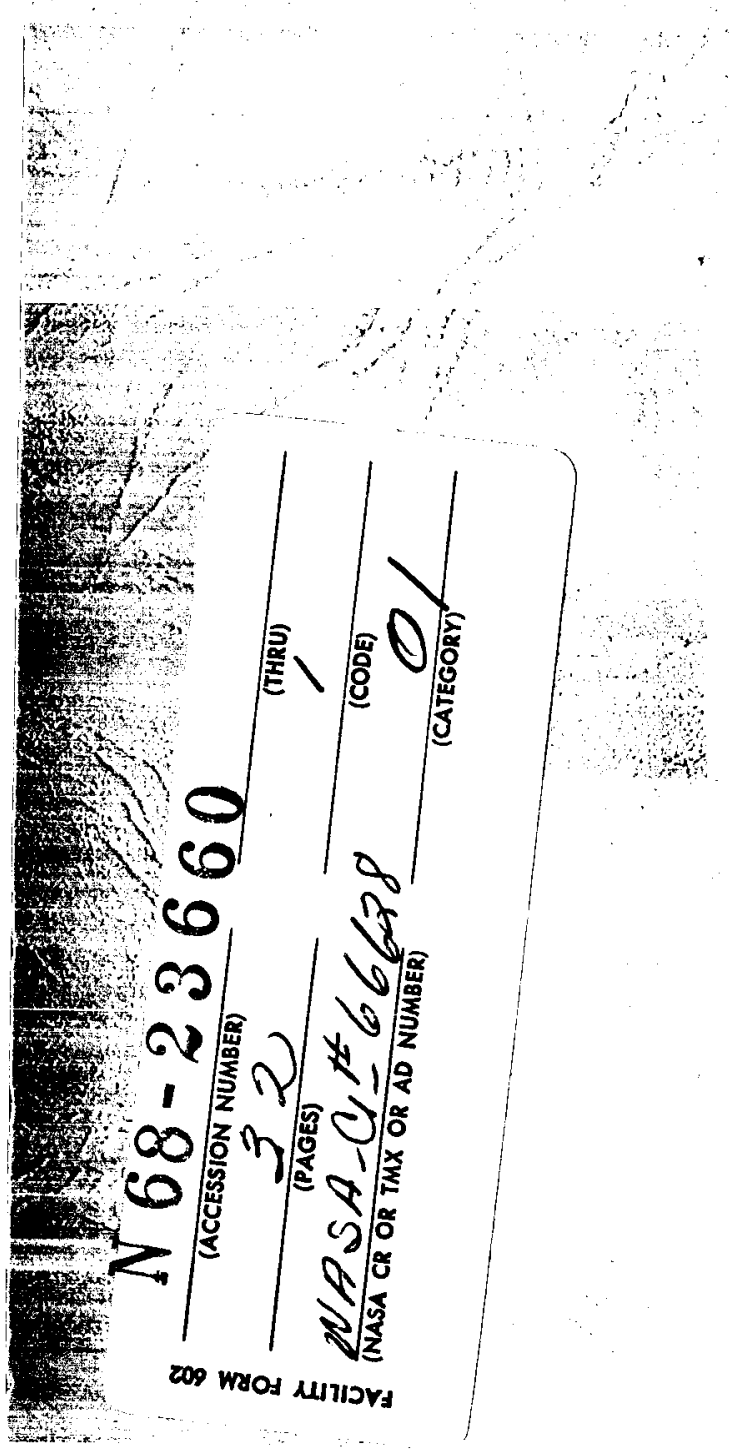

GPO PRICE $\$$
CFSTI PRICE(S) $\$$

Hard copy $(\mathrm{HC}$

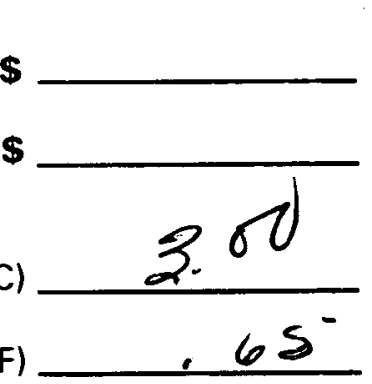

Microfiche (MF)

if 653 July 65

RICE UNIVERSITY

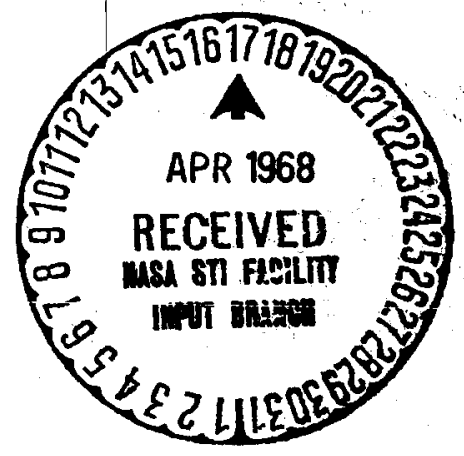

1967 


\section{Variational Approach to Conical Bodies}

\section{Having Maximum Lift-to-Drag Ratio at Hypersonic Speeds ${ }^{1}$}

$$
\text { HO- YI HUANG }{ }^{2}
$$

Abstract. An investigation of the lift-to-drag ratio $E$ attainable by a slender, conical body flying at hypersonic speeds is presented under the assumptions that the pressure distribution is modified Newtonian and the surface averaged friction coefficient is constant. The length of the body and the elongation ratio of the cross section $\alpha$ are prescribed, and the values of the free-stream dynamic pressure, the factor modifying the Newtonian pressure distribution $\mathrm{m}$, and the surface-averaged friction coefficient $\mathrm{C}_{\mathrm{f}}$ are known a priori. The indirect methods of the calculus of variations are employed, and it is found that, for any given value of the length and the elongation ratio, the optimum transversal contour is a diamond shape. As the elongation ratio increases, the maximum lift-to-drag ratio increases, tending the limiting value $\mathrm{E}=0.529 \mathrm{3} /\left(\mathrm{m} / \mathrm{C}_{\mathrm{f}}\right)$ when $\alpha \rightarrow \infty$.

\footnotetext{
${ }^{1}$ This research was supported by the NASA-Langley Research Center, Grant No. NGR-44-006-063. The author would like to express his appreciation to Dr. Angelo Miele for his encouragement and helpful discussions.

2

Graduate Student in Aero-Astronautics, Department of Mechanical and Aerospace Engineering and Materials Science, Rice University, Houston, Texas .
} 


\section{Introduction}

In Refs. 1 and 2, the lift-to-drag ratio obtainable by a slender, flat-top, homothetic body at hypersonic speeds was studied under the assumptions that the pressure distribution is modified Newtonian and the surface-averaged friction coefficient is constant. In Ref. 1, direct methods were employed, and the analysis was confined to the class of bodies whose longitudinal contour is a power-law and whose transversal contour is semielliptical or triangular. In Ref. 2 , the indirect methods of the calculus of variations were used and the longitudinal and transversal contours were optimized successively . Concerning the longitudinal contour, it was shown that the optimum solution is conical and the thickness ratio is such that the friction drag is one-third of the total drag. Concerning the transversal contour, it was shown that the optimum solution is triangular with or without a keel, depending on whether the cross-sectional elongation ratio is smaller or larger than the critical value $\alpha=4.85$.

In Ref. 3, a modification of the problem studied in Refs. 1 and 2 was investigated, that of homothetic configurations which are not necessarily flat-topped. The optimum longitudinal contour was shown to be identical with that of the previous case. Concerning the transversal contour, direct methods were employed, and a systematic analysis of a wide variety of cross sections was presented. It was found that, for a given crosssectional elongation ratio, a flat-top, triangular cross section with or without a keel is aerodynamically inferior to a flat-bottom triangle which, in turn, is less efficient than a diamond shape. The latter was found to be best among all the cross sections considered. 
Since Ref. 3 treated only particular configurations, it is the purpose of this report to extend the study to arbitrary configurations. Therefore, the extremal problem is investigated via the indirect methods of the calculus of variations. The following hypotheses are employed: (a) the body is conical; (b) a plane of symmetry exists between the left-hand and right-hand sides of the body; (c) the base plane is perpendicular to the plane of symmetry; (d) the free-stream velocity is contained in the plane of symmetry and is perpendicular to the base plane; (e) the body is slender, in the sense that the cosine squared of the angle between the local normal to the body and the undisturbed flow direction is much smaller than one; $(\mathrm{f})$ the pressure distribution is modified Newtonian, that is, the pressure coefficient is proportional to the cosine squared of the angle between the local normal to the body and the undistrubed flow direction; (g) the surface-averaged friction coefficient is constant; (h) the base drag coefficient is zero; and (i) the contribution of the tangential forces to the lift is negligible with respect to the contribution of the normal forces. 


\section{Fundamental Equations}

We consider the Cartesian coordinate system OXYZ shown in Fig. 1. The origin $\mathrm{O}$ is the apex of the body, the $\mathrm{X}$-axis is parallel to the free-stream velocity and positive toward the base, the $\mathrm{Z}$-axis is contained in the plane of symmetry and positive downward, and the $\mathrm{Y}$-axis is such that the $\mathrm{XYZ}$-system is right-handed.

We denote by $\vec{i}, \vec{j}, \vec{k}$ the unit vectors of this coordinate system, by $\vec{n}$ the unit vector normal to the infinitesimal element of wetted area $\mathrm{dS}_{\mathrm{w}}$, positive outward, and by $\vec{t}$ the unit vector which is tangent to $\mathrm{dS}_{\mathrm{w}}$ and is in the direction of the local flow after impact. Since the free-stream velocity is parallel to the $\mathrm{X}$-axis, the lift $\mathrm{L}$ and the drag $\mathrm{D}$ per unit free-stream dynamic pressure $\mathrm{q}_{\infty}$ are given by

$$
\begin{aligned}
& L / q_{\infty}=\iint_{S_{w}}\left(C_{p} \vec{n} \cdot \vec{k}-C_{f} \vec{t} \cdot \vec{k}\right) d S_{w} \\
& D / q_{\infty}=\iint_{S_{w}}\left(-C_{p} \vec{n} \cdot \vec{i}+C_{f} \vec{t} \cdot \vec{i}\right) d S_{w}
\end{aligned}
$$

where $\mathrm{C}_{\mathrm{p}}$ is the pressure coefficient and $\mathrm{C}_{\mathrm{f}}$ is the surface-averaged friction coefficient If every surface element sees the flow, the distribution of the pressure coefficient is given by

$$
\mathrm{C}_{\mathrm{p}}=2 \mathrm{~m}(\overrightarrow{\mathrm{n}} \cdot \overrightarrow{\mathrm{i}})^{2}
$$

where $\mathrm{m}$ is the factor modifying the Newtonian pressure law. Therefore, after the geometric relationship

$$
\mathrm{dS}_{\mathrm{w}}=|\overrightarrow{\mathrm{n}} \cdot \overrightarrow{\mathrm{k}}|^{-1} \mathrm{dXdY}
$$


is introduced, the aerodynamic forces can be rewritten in the form

$$
\begin{aligned}
& L / q_{\infty}=\iint_{I+I I}|\vec{n} \cdot \vec{k}|^{-1}\left[2 m(\vec{n} \cdot \vec{i})^{2}(\vec{n} \cdot \vec{k})-C_{f} \vec{t} \cdot \vec{k}\right] d X d Y \\
& D / q_{\infty}=\iint_{I+I I}|\vec{n} \cdot \vec{k}|^{-1}\left[-2 m(\vec{n} \cdot \vec{i})^{3}+C_{f} \vec{t} \cdot \vec{i}\right] d X d Y
\end{aligned}
$$

The symbols I and II refer to the upper and lower surfaces of the body, respectively. After the normal and tangent unit vectors are expressed by

$$
\begin{aligned}
\vec{n} & =\alpha \vec{i}+\beta \vec{j}+\gamma \vec{k}, & & \gamma \leq 0 \text { on I, } \gamma \geq 0 \text { on II } \\
t & =a \vec{i}+b \vec{j}+c \vec{k}, & & a \geq 0
\end{aligned}
$$

Eqs. (4) become

$$
\begin{aligned}
& L / q_{\infty}=\iint_{I+I I}|\gamma|^{-1}\left(2 m \alpha^{2} \gamma-C_{f} c\right) d X d Y \\
& D / q_{\infty}=\iint_{I+I I}|\gamma|^{-1}\left(-2 m \alpha^{3}+C_{f} a\right) d X d Y
\end{aligned}
$$

If the geometry of the body is described by the equation

$$
f(X, Y, Z)=0
$$

the normal unit vector and the gradient of the function $f$ are parallel with the implication that

$$
\alpha=\mathrm{f}_{\mathrm{X}} / \mathrm{g}, \quad B=\mathrm{f}_{\mathrm{Y}} / \mathrm{g}, \quad \gamma=\mathrm{f}_{\mathrm{Z}} / \mathrm{g}
$$

where

$$
\left.g=\sqrt{ } / f_{X}^{2}+f_{Y}^{2}+f_{Z}^{2}\right)
$$


Since the tangent vector has unit modulus, is perpendicular to $\vec{n}$, and is coplanar with $\vec{n}$ and $\vec{i}$, the equations determining the components $a, b, c$ are written as

$$
\vec{t} \cdot \vec{t}=1, \vec{t} \cdot \vec{n}=0, \vec{t} \cdot \vec{n} \times \vec{i}=0
$$

and, in explicit form, become

$$
\begin{aligned}
& a^{2}+b^{2}+c^{2}=1 \\
& \alpha a+B b+\gamma c=0 \\
& \gamma b-B c=0
\end{aligned}
$$

These equations are solved by

$$
a=\sqrt{ }\left(1-\alpha^{2}\right), b=-\alpha \beta / \sqrt{ }\left(1-\alpha^{2}\right), c=-\alpha \gamma / \sqrt{ }\left(1-\alpha^{2}\right)
$$

with the implication that

$$
a=h / g, b=-f_{X} f_{Y} / g h, c=-f_{X} f_{Z} / g h
$$

where

$$
\mathrm{h}=\sqrt{ }\left(\mathrm{f}_{\mathrm{Y}}^{2}+\mathrm{f}_{\mathrm{Z}}^{2}\right)
$$

If relationships (8) and (13) are used, the expressions (6) become

$$
\begin{aligned}
& L / q_{\infty}=\iint_{I+I I} f_{X} f_{Z}\left|f_{Z}\right|^{-1}\left(2 \mathrm{mf}_{X} / g^{2}+C_{f} / h\right) d X d Y \\
& D / q_{\infty}=\iint_{I+I I}\left|f_{Z}\right|^{-1}\left(-2 \mathrm{mf}_{X}^{3} / g^{2}+C_{f} h\right) d X d Y
\end{aligned}
$$


If the geometry of the body is expressed in the form

$$
f(X, Y, Z) \equiv \mp Z \pm Z(X, Y)=0
$$

where the upper sign applies to the upper surface I and the lower sign to the lower surface II, the following relationships hold:

$$
f_{X}= \pm Z_{X}, f_{Y}= \pm Z_{Y}, f_{Z}=\mp 1
$$

and imply that

$$
g=\sqrt{ }\left(1+Z_{X}^{2}+Z_{Y}^{2}\right), \quad h=\sqrt{ }\left(1+Z_{Y}^{2}\right)
$$

Consequently, Eqs. (15) can be rewritten as

$$
\begin{aligned}
& L / q_{\infty}=\iint_{I+I I} Z_{X}\left(\mp 2 m Z_{X} / g^{2}-C_{f} / h\right) d X d Y \\
& D / q_{\infty}=\iint_{I+\Pi}\left(\mp 2 m Z_{X}^{3} / g^{2}+C_{f} h\right) d X d Y
\end{aligned}
$$

and simplify to

$$
\begin{aligned}
& L / q_{\infty}=\iint_{I+I I}\left(\mp 2 \mathrm{mZ}_{X}^{2} / \mathrm{g}^{2}\right) d X d Y \\
& D / q_{\infty}=\iint_{I+I I}\left(\mp 2 \mathrm{mZ}_{X}^{3} / \mathrm{g}^{2}+\mathrm{C}_{\mathrm{f}} \mathrm{h}\right) \mathrm{dX} \mathrm{dY}
\end{aligned}
$$

if the contribution of the tangential forces to the lift is negligible with respect to the contribution of the normal forces.

If the body is slender in the sense of hypothesis (e), that is, 


$$
(\vec{n} \cdot \vec{i})^{2} \ll 1
$$

the use of Eqs . (5), (8), (17), and (18) leads to

$$
g=h
$$

so that Eqs . (20) are reduced to

$$
\begin{aligned}
& \mathrm{L} / \mathrm{q}_{\infty}=\iint_{\mathrm{I}+\mathrm{II}}\left[\mp 2 \mathrm{mz}_{\mathrm{X}}^{2} /\left(1+\mathrm{Z}_{\mathrm{Y}}^{2}\right)\right] \mathrm{dXdY} \\
& \mathrm{D} / \mathrm{q}_{\infty}=\iint_{\mathrm{I}+\mathrm{II}}\left[\mp 2 \mathrm{mz}_{\mathrm{X}}^{3} /\left(1+\mathrm{Z}_{\mathrm{Y}}^{2}\right)+\mathrm{C}_{\mathrm{f}^{\prime}} /\left(1+\mathrm{Z}_{\mathrm{Y}}^{2}\right)\right] \mathrm{dXdY}
\end{aligned}
$$

2.1. Conical Body. The geometry of a conical body can be expressed by the parametric equations

$$
\mathrm{Y}=(\mathrm{X} / \ell) \mathrm{Y}_{\mathrm{b}}, \quad \mathrm{Z}=(\mathrm{X} / \ell) \mathrm{Z}_{\mathrm{b}}
$$

where $\ell$ is the length and where $Y_{b}, Z_{b}$ denote the coordinates of the base contour $\mathrm{X}=\ell$. On the base, $\mathrm{Z}_{\mathrm{b}}=\mathrm{Z}_{\mathrm{b}}\left(\mathrm{Y}_{\mathrm{b}}\right)$. For the body (24), the surface integrals (23) reduce to the line integrals

$$
\begin{aligned}
& \mathrm{L} / \mathrm{q}_{\infty}=\int_{\mathrm{I}+\mathrm{II}}\left[\mp(\mathrm{m} / \mathrm{l})\left(\mathrm{Z}_{\mathrm{b}}-\mathrm{Y}_{\mathrm{b}} \dot{\mathrm{Z}}_{\mathrm{b}}\right)^{2} /\left(1+\dot{\mathrm{Z}}_{\mathrm{b}}^{2}\right)\right] \mathrm{dY_{ \textrm {b } }} \\
& \mathrm{D} / \mathrm{q}_{\infty}=\int_{\mathrm{I}+\mathrm{II}}\left[\mp\left(\mathrm{m} / \ell^{2}\right)\left(\mathrm{Z}_{\mathrm{b}}-\mathrm{Y}_{\mathrm{b}} \dot{\mathrm{Z}}_{\mathrm{b}}\right)^{3} /\left(1+\dot{\mathrm{Z}}_{\mathrm{b}}^{2}\right)+\left(\mathrm{C}_{\mathrm{f}} \ell / 2\right) /\left(1+\dot{\mathrm{Z}}_{\mathrm{b}}^{2}\right)\right] \mathrm{dY} \mathrm{y}_{\mathrm{b}}
\end{aligned}
$$

where $\dot{\mathrm{Z}}_{\mathrm{b}}=\mathrm{dZ} \mathrm{i}_{\mathrm{b}} / \mathrm{dY}_{\mathrm{b}}$. 
2.2. Dimensionless Quantities. We now define the dimensionless base

coordinates

$$
\mathrm{y}=\mathrm{Y}_{\mathrm{b}} / \ell \mathrm{k}, \quad \mathrm{z}=\mathrm{Z}_{\mathrm{b}} / \ell \mathrm{k}
$$

where the constant $k$ is given by

$$
k=\sqrt[3]{ }\left(\mathrm{C}_{\mathrm{f}} / \mathrm{m}\right)
$$

Next, we define the modified lift $\mathrm{L}_{*}$ and drag $\mathrm{D}_{*}$ as follows:

$$
\mathrm{L}_{*}=\mathrm{L} / \mathrm{q}_{\infty} \mathrm{m} \ell^{2} \mathrm{k}^{3}, \quad \mathrm{D}_{*}=\mathrm{D} / \mathrm{q}_{\infty} \mathrm{m} \ell^{2} \mathrm{k}^{4}
$$

With this understanding, Eqs . (25) can be rewritten as

$$
\begin{aligned}
& L_{*}=\int_{I+I I}\left[\mp(z-y \dot{z})^{2} /\left(1+\dot{z}^{2}\right)\right] d y \\
& D_{*}=\int_{I+I I}\left[\mp(z-y \dot{z})^{3} /\left(1+\dot{z}^{2}\right)+(1 / 2) \sqrt{ } /\left(1+\dot{z}^{2}\right)\right] d y
\end{aligned}
$$

where $\dot{z}=d z / d y$.

2.3. Remark. The Newtonian pressure law (2) is valid providing every surface element sees the flow. Therefore, the local contribution to the pressure drag must be nonnegative. This is equivalent to stating that the inequalities

$$
\mp(z-y \dot{z}) \geq 0
$$

must be satisfied at every point of the base contour. 
2.4. Lift-to-Drag Ratio. The lift-to-drag ratio is defined as

$$
E=L / D
$$

For the purposes of this paper, it is convenient to introduce the modified lift-to-drag ratio

$$
\mathrm{E}_{*}=\mathrm{Ek}
$$

and observe that, because of Eqs . (28),

$$
\mathrm{E}_{*}=\mathrm{L}_{*} / \mathrm{D}_{*}
$$




\section{Optimum Transversal Contour Problem}

Consider a conical body having the dimensionless base contour $z=z(y)$. Since the contour is symmetric with respect to the z-axis, we limit the analysis to the semiplane $y \geq 0$. Let $y_{f}$ be the abscissa of the maximum width point, the point separating the upper and lower contours, and let the base contour be described by the relations (Fig. 2)

$$
\begin{array}{ll}
\text { Upper contour } & \mathrm{z}=\mathrm{u}(\mathrm{y}) \\
\text { Lower contour } & \mathrm{z}=\mathrm{w}(\mathrm{y})
\end{array}
$$

With this understanding, Ineqs. (30) become

$$
\text { yu் }-u \geq 0, \quad y \dot{w}-w \leq 0
$$

and are equivalent to

$$
\mathrm{p}^{2}+\mathrm{u}-\mathrm{y} \dot{\mathrm{u}}=0, \quad \mathrm{q}^{2}-\mathrm{w}+\mathrm{y} \dot{\mathrm{w}}=0
$$

where $\dot{\mathrm{u}}=\mathrm{du} / \mathrm{dy}, \dot{\mathrm{w}}=\mathrm{dw} / \mathrm{dy}$ and where $\mathrm{p}, \mathrm{q}$ are real functions of $\mathrm{y}$. Because of the definitions (34) and (36) and because the body has a plane of symmetry, the modified lift and drag (29) can be rewritten in the form

$$
\begin{aligned}
& \mathrm{L}_{*}=\int_{\mathrm{y}_{\mathrm{i}}}^{\mathrm{y}_{\mathrm{f}}}\left[-2 \mathrm{p} /\left(1+\dot{\mathrm{u}}^{2}\right)+2 \mathrm{q}^{4} /\left(1+\dot{\mathrm{w}}^{2}\right)\right] \mathrm{dy} \\
& \mathrm{D}_{*}=\int_{\mathrm{y}_{\mathrm{i}}}^{\mathrm{y}} \mathrm{f}_{\left.\mathrm{f}^{6} /\left(1+\dot{\mathrm{u}}^{2}\right)+\sqrt{ }\left(1+\dot{\mathrm{u}}^{2}\right)+2 \mathrm{q}^{6} /\left(1+\dot{\mathrm{w}}^{2}\right)+\sqrt{ }\left(1+\dot{\mathrm{w}}^{2}\right)\right] \mathrm{dy}}
\end{aligned}
$$

It is now assumed that the length $l$, the factor modifying the Newtonian pressure law $m$, and the surface-averaged friction coefficient $C_{f}$ are known a priori, so that the constant $\mathrm{k}$, defined by Eq. (27), is also known a priori. With this understanding, 
the problem of maximizing the lift-to-drag ratio (31) is equivalent to maximizing the modified lift-to-drag ratio (33) subject to the definitions (37) and the differential constraints (36). Since the initial point is located in the plane of symmetry and the final point is the point of intersection of the upper and lower contours, the following boundary conditions must be considered:

$$
y_{i}=0, \quad u_{f}-w_{f}=0
$$

It can be shown that the extremal solution of the problem formulated above is a cross section of infinite width. Since this solution is unrealistic from an engineering point of view, some geometric constraint is needed. Therefore, as in Refs. 1 through 3 , we require the cross-sectional elongation ratio

$$
\alpha=y_{f} /\left(w_{i}-u_{i}\right)
$$

to be a prescribed quantity. 


\section{Necessary Conditions}

In accordance with the treatment of Refs. 4 and 5, the necessary conditions for the problem stated in the previous section are idemical with those characterizing the functional

$$
I=\int_{y_{i}}^{y_{f}}[F(y, u, \dot{u}, p, \lambda, u)+G(y, w, \dot{w}, q, \lambda, \nu)] d y
$$

where $\mathrm{F}$ and $\mathrm{G}$ denote the functions

$$
\begin{aligned}
& F=-2 p^{4} /\left(1+\dot{u}^{2}\right)-\lambda\left[2 p^{6} /\left(1+\dot{u}^{2}\right)+\sqrt{ }\left(1+\dot{u}^{2}\right)\right]-\mu\left(p^{2}+u-y \dot{u}\right) \\
& G=2 q^{4} /\left(1+\dot{w}^{2}\right)-\lambda\left[2 q^{6} /\left(1+\dot{w}^{2}\right)+\sqrt{ }\left(1+\dot{w}^{2}\right)\right]-\nu\left(q^{2}-w+y \dot{w}\right)
\end{aligned}
$$

Here, $u$ and $v$ are variable Lagrange multipliers and $\lambda$ is a constant Lagrange multiplier whose value is

$$
\lambda=\mathrm{E}_{*}
$$

From calculus of variations (see, for example, Chapter 2 of Ref. 6), it is known that the extremal arc must be a solution of the Euler equations

$$
\begin{array}{ll}
F_{p}=0, & d F_{\dot{u}} / d y-F_{u}=0 \\
G_{q}=0, & d G_{\dot{w}} / d y-G_{w}=0
\end{array}
$$

The first two equations are relevant to the upper contour, while the last two equations are relevant to the lower contour. The functions solving Eqs . (43) must satisfy the transversality condition 


$$
\left[\left(F+G-\dot{u} F_{\dot{u}}-\dot{w} G_{\dot{w}}\right) \delta y+F_{\dot{u}} \delta u+G_{\dot{w}} \delta w\right]_{\dot{i}}^{f}=0
$$

for every set of variations consistent with the boundary conditions (38) and (39), that is,

$$
\delta y_{i}=0, \quad \delta u_{f}-\delta w_{f}=0, \quad \delta y_{f}+\alpha\left(\delta u_{i}-\delta w_{i}\right)=0
$$

As a conscquence, after some rearrangement, the transversality condition (44) yields the natural boundary conditions

$$
\begin{aligned}
& \alpha\left[F+G-\dot{u} F_{\dot{u}}-\dot{w} G_{\dot{w}}\right]_{f}+\left[F_{\dot{u}}\right]_{1}=0 \\
& {\left[F_{\dot{u}}+G_{\dot{w}}\right]_{\dot{i}}=0} \\
& {\left[F_{\dot{u}}+G_{\dot{w}}\right]_{f}=0}
\end{aligned}
$$

which, together with Eqs. (38) and (39), constitute the set of boundary conditions to be satisfied by the solutions of the Euler equations (43).

Once a set of solutions satisfying the Euler equations and the boundary conditions is found, one has to verify that it yields a maximum for the functional (40). In this connection, the Weierstrass condition is of considerable assistance. It states that the inequality

$$
\Delta \mathrm{F}+\Delta \mathrm{G}-\mathrm{F}_{\dot{\mathrm{u}}} \Delta \dot{\mathrm{u}}-\mathrm{G}_{\dot{\mathrm{w}}} \Delta \dot{\mathrm{w}} \leq 0
$$

must be satisfied at every point of the maximal arc. In the above inequality, the symbols $\Delta \mathrm{F}, \Delta \mathrm{G}, \Delta \dot{\mathrm{u}}$, and $\Delta \dot{\mathrm{w}}$ are defined as

$$
\begin{aligned}
& \Delta \mathrm{F}=\mathrm{F}\left(\mathrm{y}, \mathrm{u}, \dot{\mathrm{u}}_{*}, \mathrm{p}_{*}, \lambda, u\right)-\mathrm{F}(\mathrm{y}, \mathrm{u}, \dot{\mathrm{u}}, \mathrm{p}, \lambda, \mu) \\
& \Delta \mathrm{G}=\mathrm{G}\left(\mathrm{y}, \mathrm{w}, \dot{\mathrm{w}}_{*}, \mathrm{q}_{*}, \lambda, \nu\right)-\mathrm{G}(\mathrm{y}, \mathrm{w}, \dot{\mathrm{w}}, \mathrm{q}, \lambda, \nu) \\
& \Delta \dot{\mathrm{u}}=\dot{\mathrm{u}}_{*}-\dot{\mathrm{u}} \\
& \Delta \dot{\mathrm{w}}=\dot{\mathrm{w}}_{*}-\dot{\mathrm{w}}
\end{aligned}
$$


where the unstarred quantities refer to the extremal arc and the starred quantities to the comparison arc. Note that both the quantities evaluated for the extremal arc and those evaluated for the comparison arc must be consistent with the constraints (36). Since it is possible to change the shape of the upper contour while keeping the lower contour invariant, and vice versa, the Weierstrass condition splits into separate conditions

$$
\Delta \mathrm{F}-\mathrm{F}_{\dot{\mathrm{u}}} \Delta \dot{\mathrm{u}} \leq 0, \quad \Delta \mathrm{G}-\mathrm{G}_{\dot{\mathrm{w}}} \Delta \dot{\mathrm{w}} \leq 0
$$

The first of these holds for the upper contour and the second for the lower contour. 


\section{Solution Process}

If the definition $(41-1)$ is combined with the Euler equations (43-1) and (43-2)

and the multiplier $u$ is eliminated, we see that the upper contour includes the subarcs

$$
y \dot{u}-u=0
$$

and

$$
\ddot{u}=A(y, u, \dot{u}, \lambda) / B(y, u, \dot{u}, \lambda)
$$

where

$$
\begin{aligned}
& A(y, u, \dot{u}, \lambda)=4(y \dot{u}-u)[3 \lambda(y \dot{u}-u)+2]\left(1+\dot{u}^{2}\right)^{2} \\
& B(y, u, \dot{u}, \lambda)=4(y \dot{u}-u)^{2}\left(1-3 \dot{u}^{2}\right)[\lambda(y \dot{u}-u)+1]-\lambda \sqrt{ }\left(1+\dot{u}^{2}\right)^{3} \\
& \quad+8 y \dot{u}(y \dot{u}-u)[3 \lambda(y \dot{u}-u)+2]\left(1+\dot{u}^{2}\right)-4 y^{2}[3 \lambda(y \dot{u}-u)+1]\left(1+\dot{u}^{2}\right)^{2}
\end{aligned}
$$

Analogously, if Eq. (41-2) is combined with Eqs . (43-3) and (43-4) and the multiplier $\nu$ is eliminated, we conclude that the lower contour includes the subarcs

$$
\mathrm{y} \dot{\mathrm{w}}-\mathrm{w}=0
$$

and

$$
\ddot{w}=C(y, w, \dot{w}, \lambda) / D(y, w, \dot{w}, \lambda)
$$

where

$$
\begin{aligned}
& C(y, w, \dot{w}, \lambda)=4(y \dot{w}-w)\left[3 \lambda\left(y \dot{w}^{-} w\right)+2\right]\left(1+\dot{w}^{2}\right)^{2} \\
& D(y, w, \dot{w}, \lambda)=4(y \dot{w}-w)^{2}\left(1-3 \dot{w}^{2}\right)[\lambda(y \dot{w}-w)+1]+\lambda \sqrt{ } /\left(1+\dot{w}^{2}\right)^{3} \\
& \quad+8 y \dot{w}(y \dot{w}-w)[3 \lambda(y \dot{w}-w)+2]\left(1+\dot{w}^{2}\right)-4 y^{2}[3 \lambda(y \dot{w}-w)+1]\left(1+\dot{w}^{2}\right)^{2}
\end{aligned}
$$


5.1. Remark. It can be verified that Eqs. (51) and (54) admit solutions of the form

$$
\mathrm{y} \dot{\mathrm{u}}-\mathrm{u}+\mathrm{C}_{1}=0, \quad \mathrm{yw}-\mathrm{w}+\mathrm{C}_{2}=0
$$

providing the integration constants are chosen to be

$$
\mathrm{C}_{1}=2 / 3 \lambda, \quad \mathrm{C}_{2}=2 / 3 \lambda
$$

On account of Eq. (42), the multiplier $\lambda$ is positive and, hence, the constants $C_{1}$ and $\mathrm{C}_{2}$ are positive. As a consequence, Eq. (56-1) is incompatible with Ineq. (35-1), while Eq. (56-2) is compatible with Ineq. (35-2).

5.2. Extremal Arc. The next step is to examine how the previous subarcs must be combined so as to satisfy the appropriate boundary conditions as well as the Weierstrass condition.

No clear-cut method exists for this part of the problem and a trial-and-error procedure must be employed. Laborious manipulations, omitted for the sake of brevity, show that (a) the upper contour is described by Eq. (50) and (b) the lower contour is described by Eq. (54), more specifically, by its particular solution (56-2). Therefore, in differential form, the extremal arc is given by

$$
\begin{aligned}
& \text { Upper contour } \quad y \dot{\mathrm{u}}-\mathrm{u}=0 \\
& \text { Lower contour } \quad \mathrm{y} \dot{\mathrm{w}}-\mathrm{w}=-\mathrm{C}_{2}, \quad \mathrm{C}_{2}=2 / 3 \lambda
\end{aligned}
$$

Upon integration, Eqs . (58) lead to

Upper contour

$$
\mathrm{u}=\mathrm{C}_{3} \mathrm{y}
$$

Lower contour

$$
\mathrm{w}=\mathrm{C}_{4} \mathrm{y}+\mathrm{C}_{2}, \quad \mathrm{C}_{2}=2 / 3 \lambda
$$


where $\mathrm{C}_{3}$ and $\mathrm{C}_{4}$ are constants.

In order to evaluate the constants, we invoke the prescribed boundary conditions (38) and (39) and the natural boundary conditions (46). In this connection, laborious manipulations yield the relationships

$$
\begin{aligned}
& \alpha\left(C_{4}-C_{3}\right)+1=0 \\
& C_{2}^{3}-\sqrt{ }\left(1+C_{4}^{2}\right)^{3}\left[1+\sqrt{ }\left(1+C_{3}^{2}\right) / \sqrt{ }\left(1+C_{4}^{2}\right)\right]=0 \\
& 2 C_{2}^{3}+\sqrt{ }\left(1+C_{4}^{2}\right)^{3}\left[1+C_{3} \sqrt{ }\left(1+C_{4}^{2}\right) / C_{4} \sqrt{ }\left(1+C_{3}^{2}\right)\right]=0
\end{aligned}
$$

which admit solutions of the form

$$
\mathrm{C}_{2}=\mathrm{C}_{2}(\alpha), \quad \mathrm{C}_{3}=\mathrm{C}_{3}(\alpha), \quad \mathrm{C}_{4}=\mathrm{C}_{4}(\alpha)
$$

Once the constants are known, the terminal coordinates of the upper and lower contours can be calculated with the relations

$$
\begin{aligned}
& \mathrm{y}_{\mathrm{i}}=\mathrm{u}_{\mathrm{i}}=0, \quad \mathrm{w}_{\mathrm{i}}=\mathrm{C}_{2}(\alpha) \\
& \mathrm{y}_{\mathrm{f}}=\alpha \mathrm{C}_{2}(\alpha), \quad \mathrm{u}_{\mathrm{f}}=\mathrm{w}_{\mathrm{f}}=\mathrm{C}_{2}(\alpha)\left[1+\alpha \mathrm{C}_{4}(\alpha)\right]
\end{aligned}
$$

Next, from (59-2), we see that the Lagrange multiplier is given by

$$
\lambda=2 / 3 \mathrm{C}_{2}(\alpha)
$$

Upon substituting Eqs . (59) into the expressions for the lift and the drag, integrating, and accounting for Eqs. (60-2) and (62), we see that the modified lift-to-drag ratio is 
given by

$$
E_{*}=2 / 3 C_{2}(x)
$$

Clearly, Eqs. (63) and (64) are compatible with Eq. (42). 


\section{Discussion and Conclusions}

In the previous sections, the optimization of the lift-to-drag ratio of a slender, conical body flying at hypersonic speeds is presented under the assumptions that the pressure distribution is modified Newtonian and the surface-averaged friction coefficient is constant. The length of the body and the elongation ratio of the cross section are prescribed, and the values of the free-stream dynamic pressure, the factor modifying the Newtonian pressure distribution, and the surface-averaged friction coefficient are known a priori. The indirect methods of the calculus of variations are employed, and it is found that, for any given value of the length and the elongation ratio $\alpha$, the optimum transversal contour is a diamond shape (Fig. 3 ). At every point of this diamond shape, the Weierstrass condition is satisfied.

The results are summarized in Figs. 4 through 7, which present the functions

$$
y_{f}=y_{f}(\alpha), \quad w_{i}=w_{i}(\alpha), \quad w_{f}=w_{f}(\alpha), \quad E_{*}=E_{*}(\alpha)
$$

As the elongation ratio increases, the modified thickness ratio $\mathrm{w}_{\mathrm{i}}$ decreases and the modified lift-to-drag ratio $\mathrm{E}_{*}$ increases. For large elongation ratios (that is, winglike configurations), the following limiting values are approached:

$$
\lim _{\alpha \rightarrow \infty} \mathrm{w}_{i}=\sqrt[3]{2}=1.26, \quad \lim _{\alpha \rightarrow \infty} \mathrm{E}_{*}=2 / 3 \sqrt[3]{2}=0.529
$$

In closing, the following remarks are pertinent: 
(a) For comparison purposes, several arbitrary cross sections have been analyzed (Ref. 3): triangle, semiellipse, rectangle, trapezoid, bitrapezoid, triangle with a keel, caret shape, and inverted caret shape. The analysis shows that, for any cross-sectional elongation ratio $\alpha$, all of these shapes are aerodynamically inferior to the diamond shape, that is, they exhibit a lower lift-to-drag ratio.

(b) Among the previous comparison shapes, the flat-bottom triangle is the best and has a lift-to-drag ratio only slightly lower than that of the diamond shape.

(c) The reason for the excellent performance of the flat-bottom triangle is that its geometry closely approximates that of the diamond shape. The lower contour of the diamond shape is rather flat, more specifically, its lateral inclination varies between 0 and 7.3 degrees, depending on the elongation ratio $\alpha$.

(d) The wind-tunnel tests performed in Refs. 7 and 8 at $\mathrm{M}=6.9$ and $\mathrm{M}=10$ have shown that the flat-bottom triangle is aerodynamically superior to the semiellipse, the rectangle, and the trapezoid. The theoretical explanation of this result is that given in point (c). 


\section{References}

1. MIELE, A., Lift-to-Drag Ratios of Slender Bodies at Hypersonic Speeds, Journal of the Astronautical Sciences, Vol. 13, No. 1, 1966.

2. MIELE, A., HULL, D.G., and BROWN, S.L., Maximum Lift-to-Drag Ratio of a Slender, Flat-Top, Hypersonic Body, Astronautica Acta, Vol. 13, No. 2, 1967.

3. MIELE, A., and HUANG, H.Y., Engineering Considerations on the Transversal Contour of a Lifting Body, Journal of the Astronautical Sciences, Vol. 14, No. 6, 1967.

4. MIELE, A., The Extremization of Products of Powers of Functionals and Its Application to Aerodynamics, Astronautica Acta, Vol. 12, No. 1, 1966.

5. MIELE, A., On the Minimization of the Product of the Powers of Several Integrals, Journal of Optimization Theory and Applications, Vol. 1, No. 1, 1967.

6. MIELE, A., Editor, Theory of Optimum Aerodynamic Shapes, Academic Press, New York, 1965.

7. WHITEHEAD, A.H., Jr., Effect of Body Cross Section and Width-Height $\underline{\text { Ratio on Performance of Bodies and Delta-Wing-Body Combinations at }}$ Mach 6.9, NASA TN No. D-2886, 1966.

8. SPENCER, B., Jr., and FOX, C.H., Jr., Experimental Study of Optimum Body Shapes at Hypersonic Speeds, Journal of the Astronautical Sciences, Vol. 14, No. $5,1967$. 


\section{LIST OF CAPTIONS}

Fig. 1 Coordinate system.

Fig. 2 Base contour.

Fig. 3 Optimum transversal contour .

Fig. 4 Optimum value of $y_{f}$.

Fig. 5 Optimum thickness ratio.

Fig. 6 Optimum value of $\mathrm{w}_{\mathrm{f}}$.

Fig. 7 Maximum lift-to-drag ratio. 


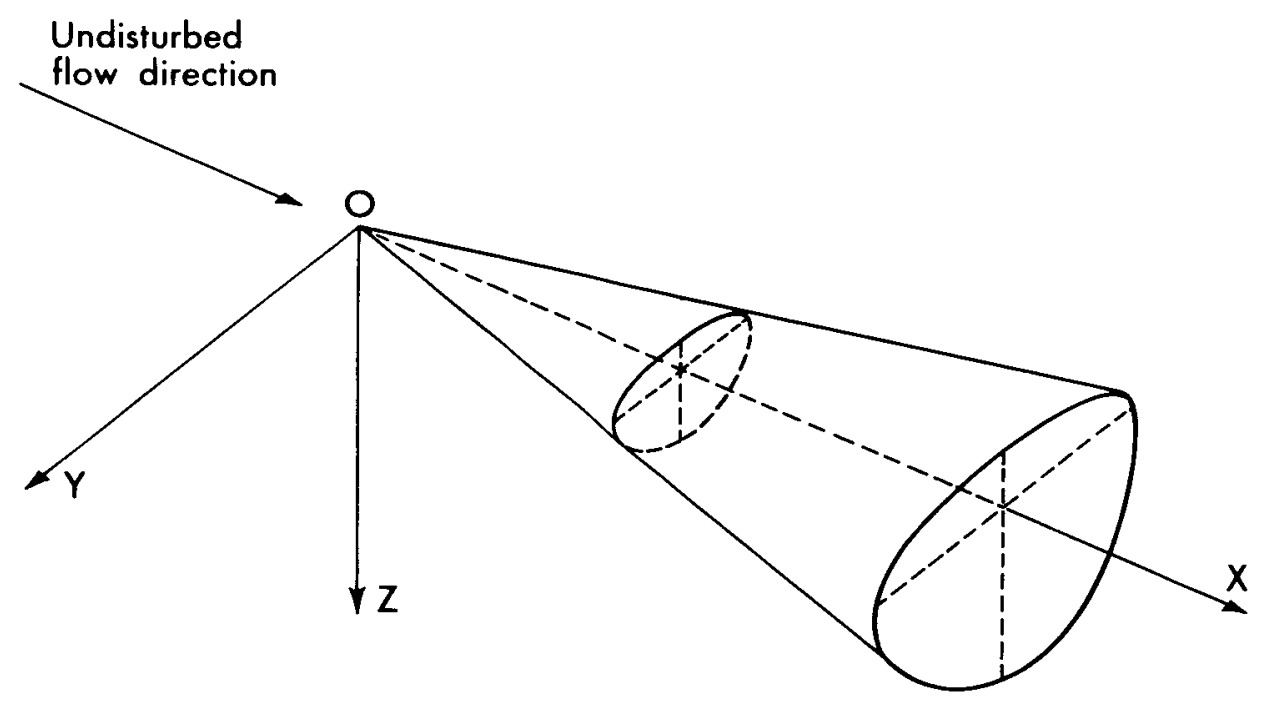

Fig. 1 Coordinate system. 


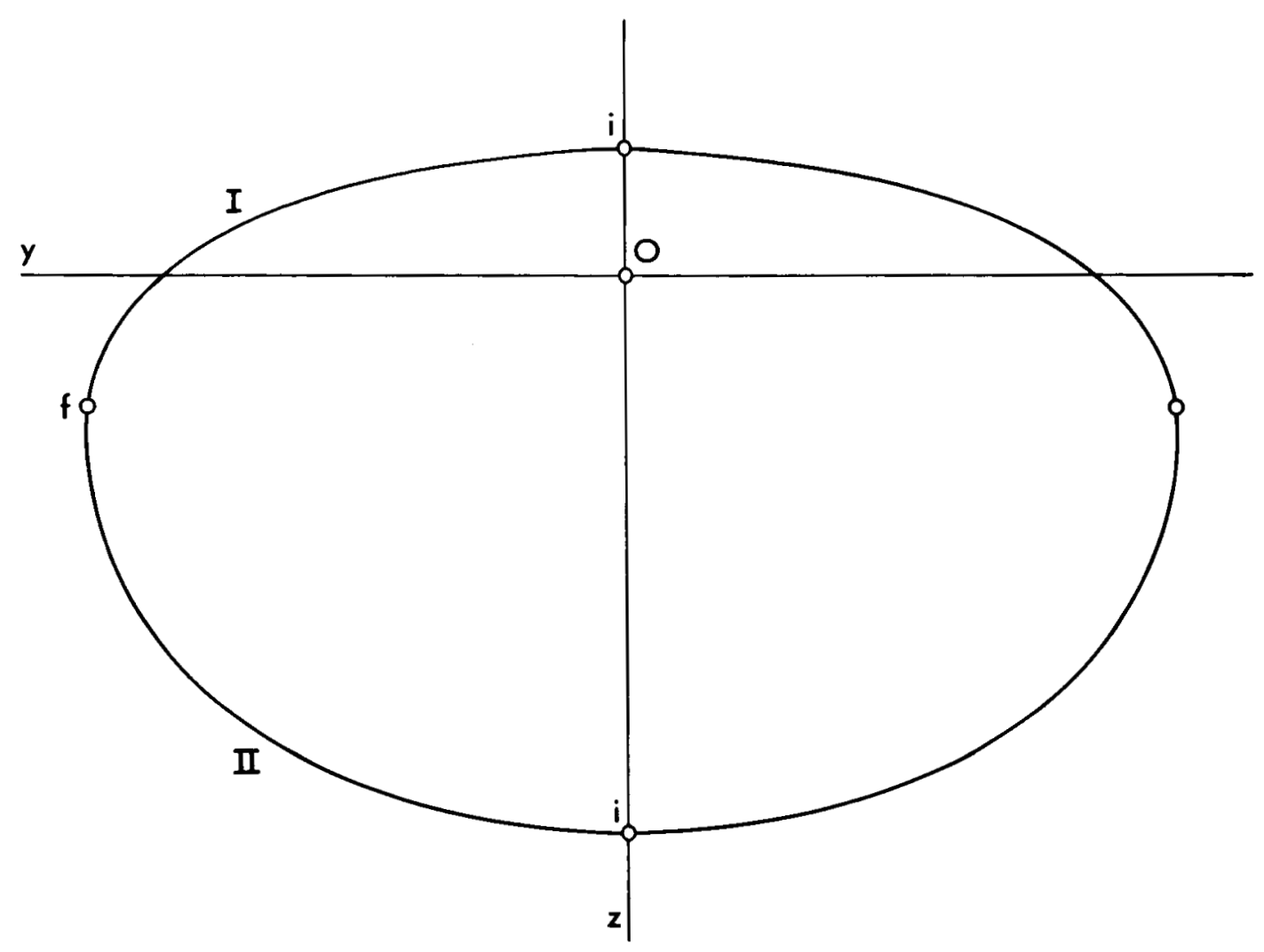

Fig. 2 Base contour.

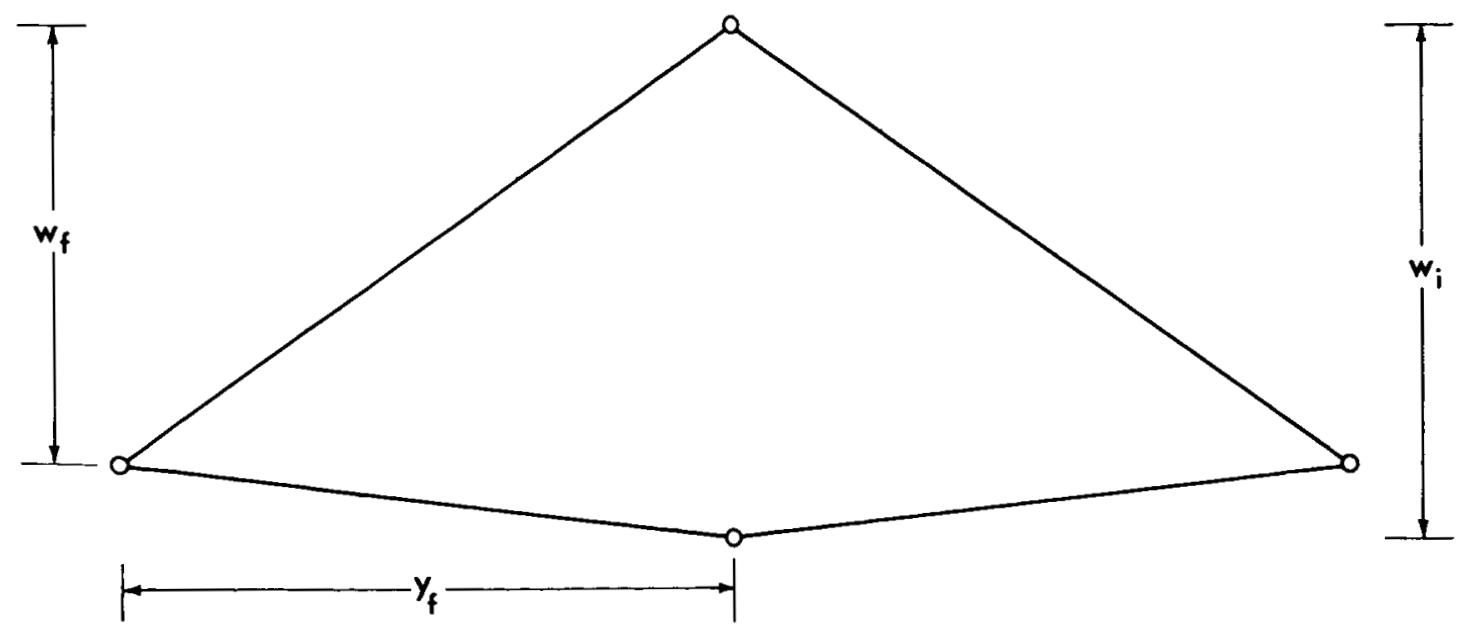

Fig. 3 Optimum transversal contour. 


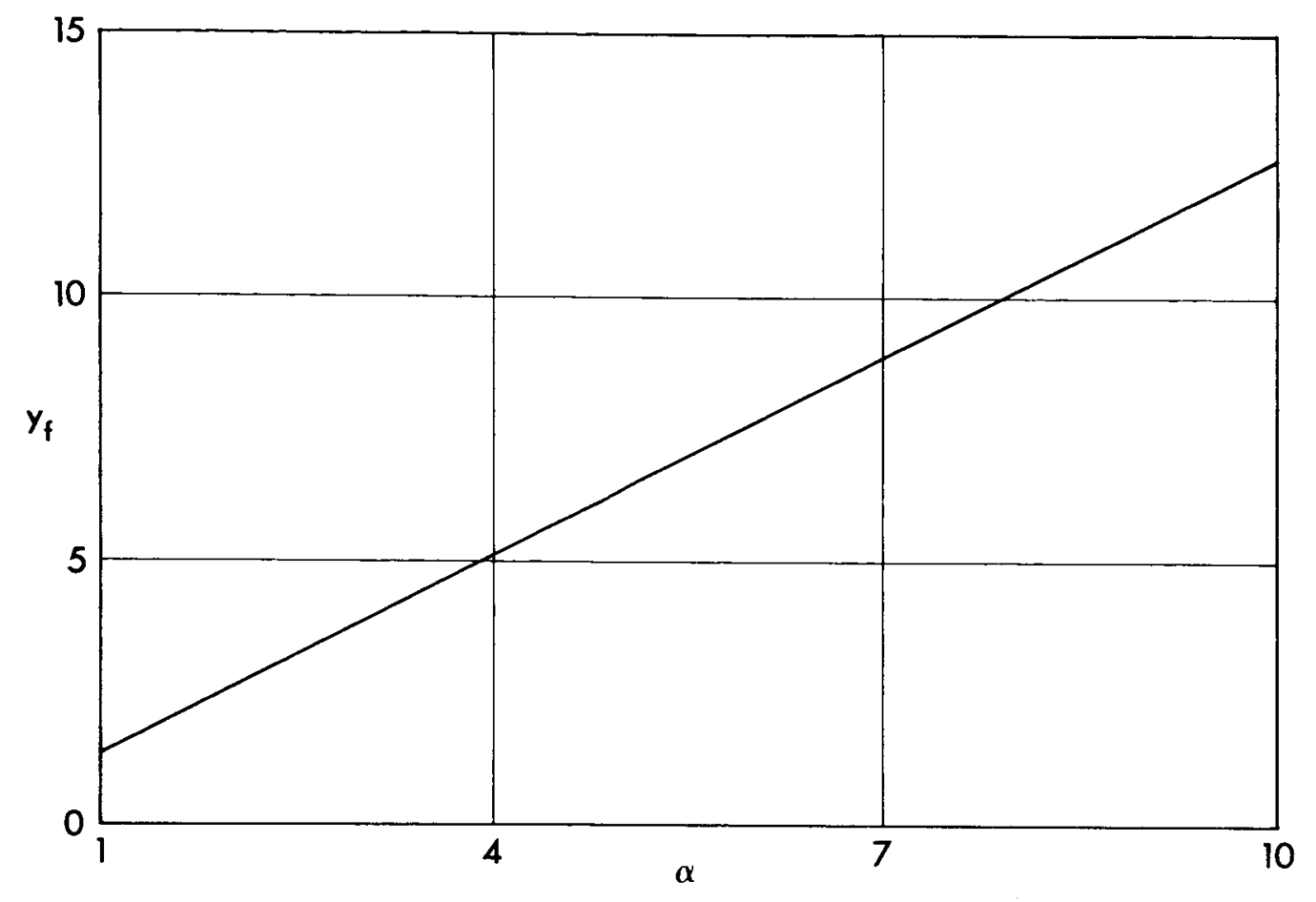

Fig. 4 Optimum value of $y_{\mathrm{f}}$.

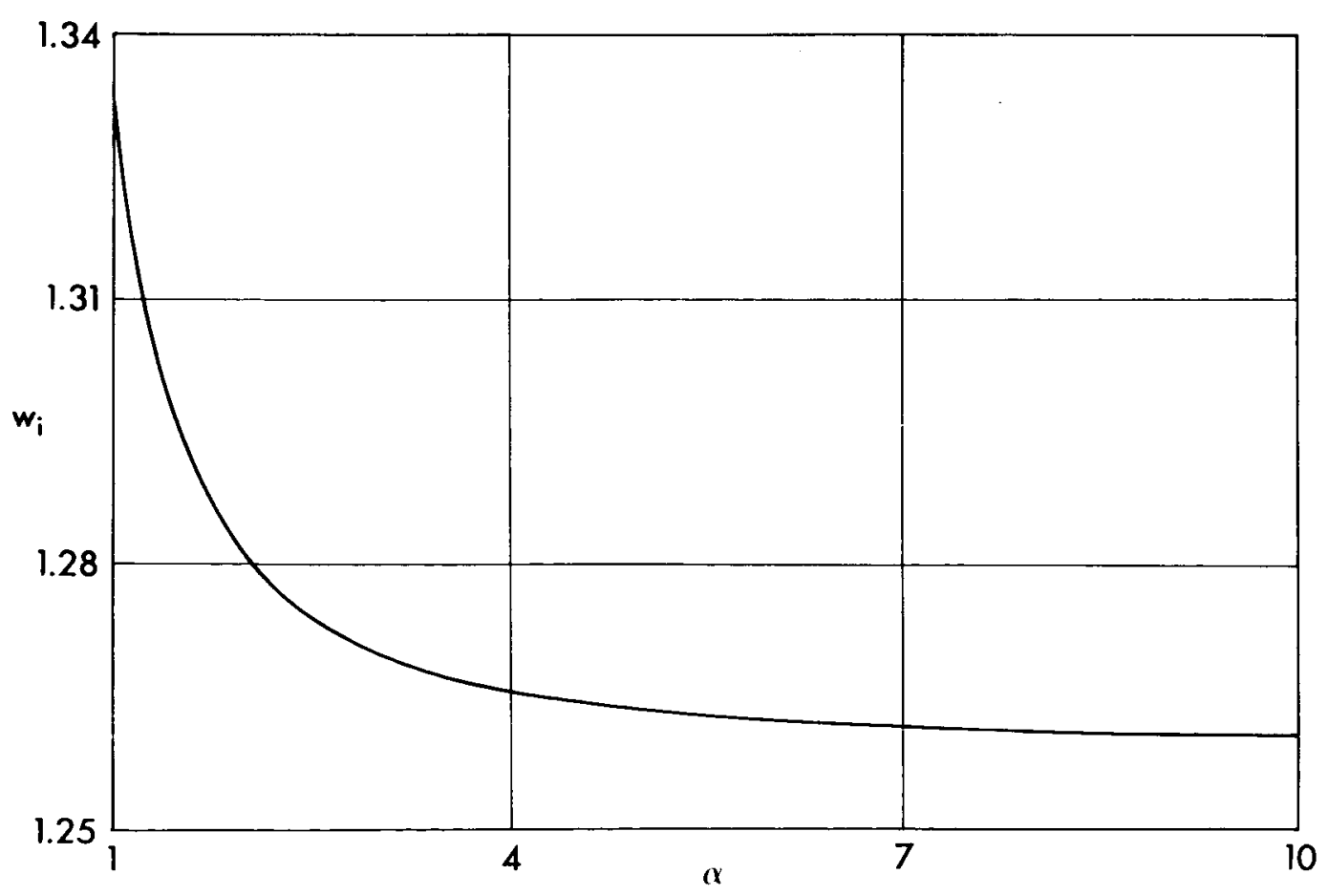

Fig. 5 Optimum thickness ratio. 


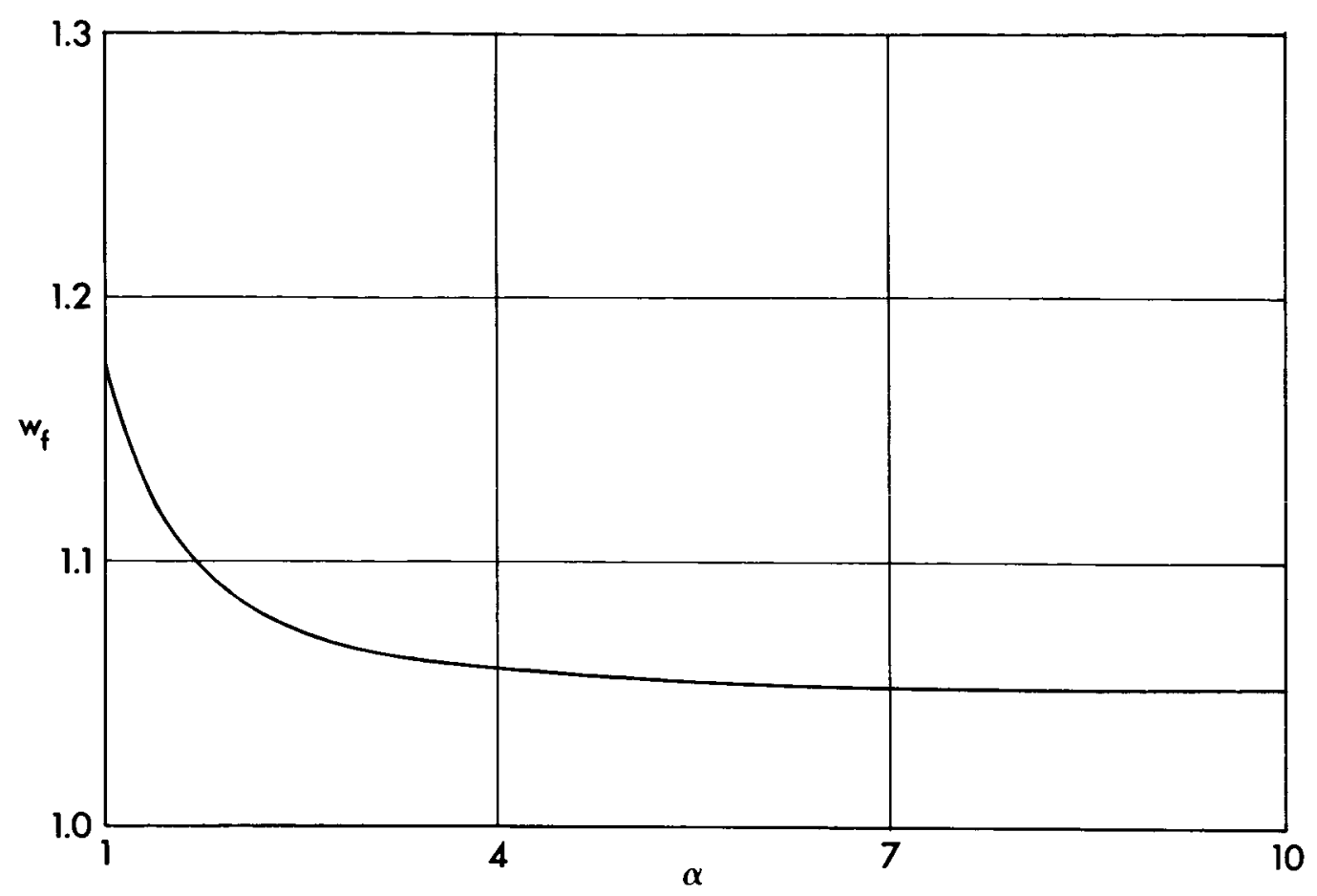

Fig. 6 Optimum value of $w_{f}$.

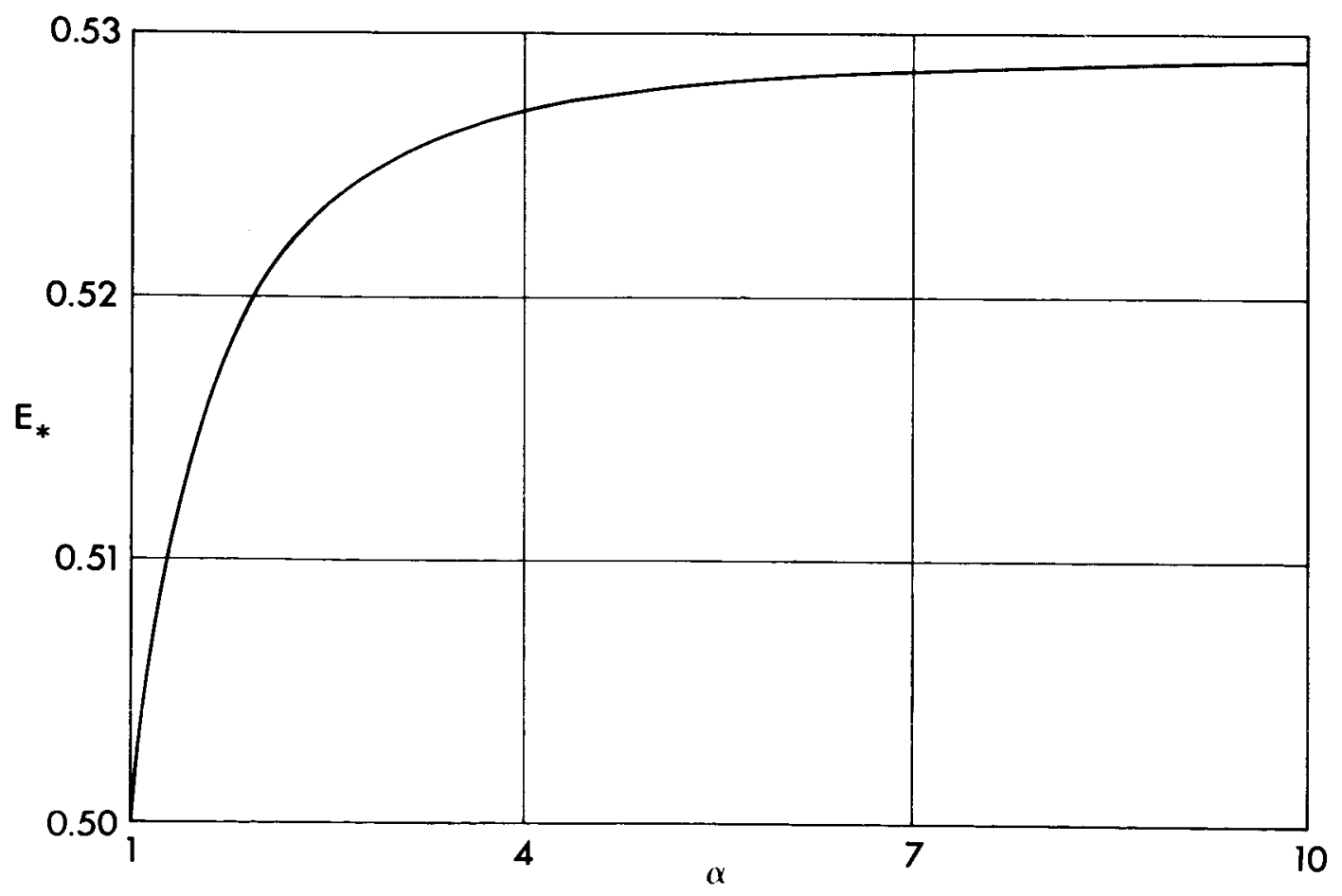

Fig. 7 Maximum lift-to-drag ratio. 